\title{
Deadzone-Quadratic Penalty Function for Predictive Extended Cruise Control with Experimental Validation
}

\author{
Seyed Amin Sajadi-Alamdari ${ }^{1}$, Holger Voos ${ }^{1}$, and Mohamed Darouach ${ }^{2}$ \\ 1 Interdisciplinary Centre for Security, Reliability and Trust (SnT), University of Luxembourg, \\ 6 rue Coudenhove-Kalergi, L-1359 Luxembourg City, Luxembourg. \\ e-mail: \{amin.sajadi, holger.voos\}@uni.lu, \\ WWW home page: https://www.uni.lu \\ 2 Centre de Recherche en Automatique de Nancy (CRAN) UMR-CNRS 7039, Université de \\ Lorraine, IUT de Longwy, 186 rue de Lorraine, F-54400 Cosnes et Romain, France. \\ e-mail: mohamed. darouacheuniv-lorraine.fr
}

\begin{abstract}
Battery Electric Vehicles have high potentials for the modern transportations, however, they are facing limited cruising range. To address this limitation, we present a semi-autonomous ecological driver assistance system to regulate the velocity with energy-efficient techniques. The main contribution of this paper is the design of a real-time nonlinear receding horizon optimal controller to plan the online cost-effective cruising velocity. Instead of conventional $\ell_{2}$-norms, a deadzone-quadratic penalty function for the nonlinear model predictive controller is proposed. Obtained field experimental results demonstrate the effectiveness of the proposed method for a semi-autonomous electric vehicle in terms of real-time energy-efficient velocity regulation and constraints satisfaction.
\end{abstract}

Keywords: Deadzone Penalty Function, Nonlinear Model Predictive Control, Ecological Advanced Driver Assistance System, Electric Vehicles

\section{Introduction}

Battery Electric Vehicle (BEV) has one of the most promising powertrain technology for the predictable future transportations [1]. However, the BEVs have limited onboard energy capacity, which limits their cruising range on a single charge. Several methods have been developed to extend the cruising range such as the Ecological Advanced Driver Assistance Systems (Eco-ADAS) with anticipated driving style. For the automotive systems, receding horizon optimal control also known as Model Predictive Control (MPC) has been an attractive approach. In MPC, an Optimal Control Problem (OCP) is solved repeatedly in a receding horizon principle. The first element in a sequence of finite control actions is applied to the system at each sampling time.

The well-established modern Cruise Control (CC) systems automate the throttle and brake control of the vehicle to retain the pre-set longitudinal velocity. Several works of literature may be founded, such as [2], where a sequential optimisation approach was presented for connected CC system. In [3] and [4], an energy-efficient linear MPC that use the energy consumption map of a BEV was established. Despite the effectiveness of the linear MPCs to some extent, they have limitations. In order to improve the performance specifications, Nonlinear MPC (NMPC) is distinguished by the use of nonlinear 
system models in the OCP. An instance work of the NMPC for the Eco-CC system considering up-down road slopes with an Internal Combustion Engine (ICE) fuel consumption model was presented in [5]. An Extended Eco-CC (Ext-Eco-CC) system that considers further road curves and traffic speed limit areas for the BEVs was introduced in [6]. Comparison and assessment of energy consumption models, cost functions, and solution methods of the Eco-CC system for passenger vehicles were reviewed in [7] and [8]. Considering the general class of (residual) penalty functions used in the NMPC, the $\ell_{2}$-norm is preferred in practice due to its efficiency in implementation. The quadratic penalty function yields least-square or Euclidean norm approximation [9]. The $\ell_{2}$-norm is preferred for energy-efficiency applications. However, the NMPC based on $\ell_{2}$-norm associated to states may also lead to aggressive system behaviour [10]. As an alternative, a systematic way of dealing with large state residuals based on Huber function was proposed in [10]. The Huber function, $\phi_{M}(x)$ is equivalent to a $\ell_{2}$-norm within the region $[-M, M]$ and to a $\ell_{1}$-norm outside. The $\ell_{1}$-norm is preferred for robust regulations where the absolute value penalty function yields $\ell_{1}$-norm approximation. Thus, the sensitivity to outliers or large residuals is lower than the $\ell_{2}$-norm [9].

Although most of the mentioned NMPCs are based on agile and intuitive set-point tracking, this may not be a suitable strategy for the energy-efficient state regulation. One of the main reason for high energy consumption of the system is strict achieving and tracking the set-point. In this paper, we propose a deadzone-quadratic and deadzonelinear penalty functions that have the advantages of $\ell_{2}$ and $\ell_{1}$-norms respectively. This method preserves the energy-efficient behaviour within the desired operating zone. The main idea of the deadzone-quadratic penalty function is to assess low penalty or insensitivity for residuals smaller than the deadzone width and quadratic or linear penalty for bigger residuals. This motivates to find a tradeoff between the agile set-point tracking and energy-efficient strategy.

The main contribution of this work is to design a real-time NMPC with deadzonequadratic penalty function to enhance the Ext-Eco-CC system for the BEVs. For this purpose, the components are considered to develop a system model. First, the BEV longitudinal dynamics, its energy consumption, as well as road geometry and traffic sign information are modelled in a reasonably accurate framework. Second, a real-time nonlinear receding horizon optimal controller is designed to plan the online cost-effective cruising velocity. Then, the NMPC takes advantage of a convex deadzone-quadratic penalty function for velocity tracking within desired reference zone. Finally, the performance of the proposed concept is evaluated in terms of energy-efficient velocity regulation and constraints fulfilment.

The remainder of this paper is structured as follows: The system model is introduced in Section 2. The NMPC formulation with deadzone-quadratic penalty function is presented in Section 3. Section 4 includes field experimental validation of the proposed concept, followed by the conclusion and future work in Section 5 .

\section{Notation}

Throughout this paper, $\mathbb{R}^{n}$ denotes the $\mathrm{n}$-dimensional Euclidean space. $\mathbb{R}_{+}:=[0, \infty)$. $\mathbb{N}=\{1,2, \ldots\}$ is set of natural numbers. $\mathbb{N}_{+}:=\mathbb{N} \cup\{0\}$ and $\mathbb{Z}_{[a, b]}:=\{a, a+1, \ldots, b\}$ is set of integers from $a$ to $b$. 


\section{System Model}

Safe and energy-efficient velocity profile identification has a significant improvement on extending the cruising range of a BEV. The semi-autonomous BEV concept that extends the functionalities of an Eco-CC system is presented in Fig. 1. Similar to the

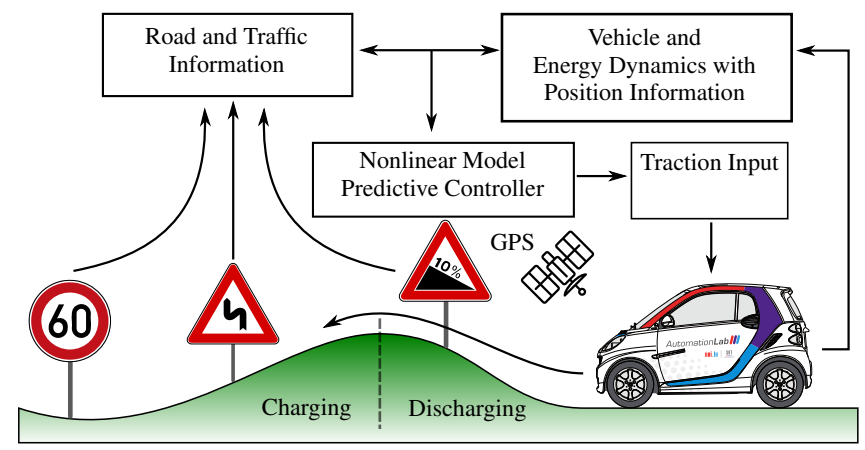

Fig. 1: Semi-autonomous BEV Ext-Eco-CC system

modern CC systems, the driver pre-sets the desired velocity. The Semi-autonomous ExtEco-CC system predictively regulates the velocity with respect to the longitudinal motion of the vehicle, its energy consumption dynamics, road geometric navigation data, and traffic sign information. While the driver handles the steering control of the vehicle, this system should plan a proper energy-efficient cruising velocity profile autonomously for the entire trip without requiring the driver interventions. For more details about the proposed Ext-Eco-CC system, see [6].

\subsection{Vehicle and Energy Dynamics}

The position $(s)$ and velocity $(v)$ along the longitudinal motion of the BEV can be expressed by Newton's second law of motion, which it is assumed to be a point mass at the centre of gravity as follows:

$$
\begin{aligned}
& \dot{s}=v, \\
& \dot{v}=\left(F_{\text {trac }}-F_{\text {res }}\right) / M,
\end{aligned}
$$

where $M, F_{\text {trac }}(t)$, and $F_{\text {res }}(t)$ are equivalent mass of the vehicle, traction force, and total motion resistive forces, respectively [1]. The traction force (throttle and brake pedals) depends on the equivalent mass and control input as $F_{\text {trac }}(t):=M u(t)$. The control input is bounded $\left(u_{\min }(v) \leq u(t) \leq u_{\max }(v)\right)$ by the physical limits of the traction force the wheel-road contact can support without slip. The main total resistive force including aerodynamic drag, gradient, and rolling resistance forces represented by:

$$
F_{r e s}=\frac{1}{2} \rho A_{f} C_{D} v^{2}+M g \sin (\theta(s))+C_{r r}(v) M g \cos (\theta(s)),
$$


where $\rho, A_{f}, C_{D}, g, \theta(s)$, and $C_{r r}(v)$, are the air density, the vehicle frontal area, the aerodynamic drag coefficient, the gravitational acceleration, the road slope angle as a function of the host vehicle position, and the velocity dependent rolling resistance coefficient, subsequently. The rolling resistance coefficient for passenger vehicles on a concrete road can be approximated as $C_{r r}(v)=0.01(1+v / 576)$ [1].

Energy consumption of a BEV depends on a number of factors including driven velocity, acceleration profile, geometric characteristics of roads, and traffic situations. For a given velocity at a given traction force, the operating point of the electric machine and the related power consumption or regeneration could be determined [6]. The energy consumption during cruising at constant speed is equal to the resistive power. This can be approximated through the curve-fit process with measurement data by a polynomial of velocity as $f_{\text {cruise }}=b_{3} v^{3}+b_{2} v^{2}+b_{1} v+b_{0}$ and acceleration as $f_{a}=a_{2} u^{2}+a_{1} u+a_{0}$. Therefore, at any given velocity and control input, a linear relation of the traction powerto-mass ratio can describe the energy consumption of the BEV as:

$$
\dot{e}=f_{a}\left(p_{\text {trac }} / M\right)+f_{\text {cruise }},
$$

where $p_{\text {trac }}=F_{\text {trac }} v$, denotes the traction power. This model is capable of capturing the energy consumption of a BEV including the regenerative braking for the full-range of velocity and the control input (for more details, see [6]).

\subsection{Road Geometry and Traffic Model}

Road geometries and traffic information have favourable advantages for the ADAS safety and energy management applications [11]. In [6], the road slopes, road curves, and traffic speed limit zone data are modelled as continuous and differentiable functions. In that method, the road slope profile $\left(f_{s l}(\theta(s))\right)$ is proposed to be the sum of quadratic functions of the vehicle position representing each road segments slope data as follows:

$$
f_{s l p}(\theta(s)):=\sum_{n=1}^{N_{s g n}} H_{\left(s-s_{n-1}\right)}^{n}\left(a_{n} s^{2}+b_{n} s+c_{n}\right) H_{\left(s-s_{n}\right)}^{n},
$$

where $N_{s g m}$ is the number of road segments, $H_{\left(s-s_{n-1}\right)}^{n}$ and $H_{\left(s-s_{n}\right)}^{n}$ are hyper-functions of the $n^{\text {th }}$ road segment. These functions represent the data points in each segment of the road utilising hyper-function concept to interconnect the estimated segments of the road at the boundaries positions, $s_{n-1}$ and $s_{n}$.

The road curves and traffic speed limits profiles are modelled in a similar way [6]. The simple curve is used to express the total absolute road curve profile $\left(f_{c r v}(\delta(s))\right)$ which is defined as:

$$
f_{c r v}(\delta(s)):=\sum_{n=1}^{N_{c r v}} H_{\left(s-s_{e n t}\right)}^{n}\left|\frac{1}{R_{c r v_{n}}(s)}\right| H_{\left(s-s_{e x t}\right)}^{n},
$$

where $N_{c r v}$ is the number of road curves, and $R_{c r v_{n}}$ is the radius of a circle valid for the curve's arc length with two position points, $s_{\text {ent }}$ and $s_{\text {ext }}$, at the respective entrance and exit positions. Furthermore, the traffic speed limit profile $\left(f_{l m t}(s)\right)$ can be modelled as:

$$
f_{l m t}(s):=\sum_{n=1}^{N_{l m t}} H_{\left(s-s_{s t r}\right)}^{n}\left(v_{l m t}-v_{\max }\right) H_{\left(s-s_{\text {end }}\right)}^{n}+v_{\max },
$$


where $N_{l m t}$ is the number of speed limit zones, and $v_{l m t}$ is the specified speed limit value at positions starts from $s_{s t r}$ up to the end of the zone $s_{\text {end }}$. The $v_{\max }$ is the maximum speed value of the electric vehicle (for more details, see [6]).

\section{Optimal Control \& Penalty Functions}

For the sake of completeness, a general NMPC formulation will be reviewed. Next, the deadzone-quadratic and deadzone-linear penalty functions will be introduced.

\subsection{Nonlinear Model Predictive Control}

Consider a general discrete-time system:

$$
x_{t+1}=f\left(x_{t}, u_{t}\right)
$$

where $t \in \mathbb{N}_{+} ; x_{t} \in \mathbb{R}^{n_{x}}$ is the system states vector, and $u_{t} \in \mathbb{U} \subset \mathbb{R}^{n_{u}}$ is a non-empty measurable set for the inputs. The $f(\cdot)$ is nonlinear Borel-measurable vector of functions that describes the system dynamics. Let $N \in \mathbb{N}$ be the both state and control prediction horizon. Define an $N$-stage feedback control policy as:

$$
\boldsymbol{\pi}:=\left\{\pi_{0}(\cdot), \pi_{1}(\cdot), \ldots, \pi_{N-1}(\cdot)\right\}
$$

where the Borel-measurable function $\pi_{i}(\cdot): \mathbb{R}^{(i+1) n_{x}} \rightarrow \mathbb{U}$, for all $i=0, \ldots, N-1$ is a general state feedback control law. The control input $u_{i}$ is selected as the feedback control law $u_{i}=\pi_{i}(\cdot)$ at the $i^{t h}$ stage of the control policy. In receding horizon optimal control, the cost function of an OCP is commonly defined as:

$$
V_{N}\left(x_{t}, \boldsymbol{\pi}\right):=\sum_{i=0}^{N-1} J_{c}\left(\hat{x}_{i}, u_{i}\right)+J_{f}\left(\hat{x}_{N}\right),
$$

where $J_{c}: \mathbb{R}^{n_{x}} \times \mathbb{U} \rightarrow \mathbb{R}_{+}$and $J_{f}: \mathbb{R}^{n_{x}} \rightarrow \mathbb{R}_{+}$are the cost-per-stage function and the final cost function, respectively, and $\hat{x}_{i}$ denotes the predicted states at time $i$ given the initial states $\hat{x}_{0}=x_{t}$, and control law $\left\{\pi_{i}(\cdot)\right\}_{i=0}^{N-1}$.

Using the cost function (10), the OCP for (8) is formulated as follows:

$$
V_{N}^{*}\left(x_{t}\right):=\underset{\pi}{\operatorname{minimise}} \quad V_{N}\left(x_{t}, \boldsymbol{\pi}\right)
$$

subject to:

$$
\begin{array}{ll}
\hat{x}_{i+1}=f\left(\hat{x}_{i}, \pi_{i}\right), & \text { for all } i \in \mathbb{Z}_{[0, N-1]}, \\
\pi_{i}(\cdot) \in U, & \text { for all } i \in \mathbb{Z}_{[0, N-1]}, \\
g_{j}\left(\hat{x}_{i}\right) \leq 0, & \text { for all } j=1, \ldots, s, \text { and } i \in \mathbb{Z}_{[0, N-1]}, \\
\hat{x}_{0}=x_{t}, &
\end{array}
$$

where $V_{N}^{*}\left(x_{t}\right)$ denotes the optimal value function under the optimal control policy $\boldsymbol{\pi}^{*}$. The inequality state constraints are denoted by $g_{j}\left(\hat{x}_{i}\right)$ that are required to be fulfilled. The OCP in receding horizon principle involves applying the first element of the control action sequence $u_{t}=\pi_{0}^{*}(\cdot)$ repeatedly to the system at each sampling time. For more details and the first-order necessary conditions for a solution of the OCP see e.g. [9] and [12]. 


\subsection{Deadzone Penalty Functions}

In many practical NMPC applications considering the energy-efficiency, it is desirable to reach a region of reference set-points with relatively low-cost value rather than costly but accurate and agile set-point tracking. This could be accomplished using a nonnegative and symmetric deadzone-quadratic penalty function such as:

$$
\phi_{q}(x):= \begin{cases}0 & :|x| \leq z \\ x^{2}-z^{2} & :|x|>z\end{cases}
$$

where $z$ is the edge of free zone that no penalty is assessed if $|x| \leq z$. The $\phi_{q}(\cdot)$ function agrees with least-square for any residual outside of the zone width. In other words, the residuals smaller than the zone width are ignored which lead to low-cost function value.

In a case of energy-efficient robust regulations, deadzone-linear penalty function agrees with absolute value for the residual outside of the zone width as follows:

$$
\phi_{l}(x):= \begin{cases}0 & :|x| \leq z \\ |x|-z & :|x|>z\end{cases}
$$

Unfortunately, these deadzone penalty functions are not convex which lead to a challenging OCPs. However, a smooth approximation of deadzone penalty function may address the challenge.

In this paper, a deadzone penalty function based on softplus rectifier is proposed. The softplus is an approximation to the activation function so-called Rectified Linear Unit (ReLU) which is mostly utilised in the deep neural networks [13]. The proposed deadzone-linear penalty function is a combination of the two softplus as follows:

$$
\psi_{l}(x):=\ln (1+\exp (x-z))+\ln (1+\exp (-x-z)) .
$$

The $\psi_{l}(x)$ have advantages such as being a convex function with efficient computation and gradient propagation [14]. The gradient of the deadzone-linear penalty function is a combination of two sigmoid functions as follows:

$$
\frac{d \psi_{l}(x)}{d x}=\frac{\exp (x-z)}{1+\exp (x-z)}-\frac{\exp (-x-z)}{1+\exp (-x-z)} .
$$

Similar to $\psi_{l}(x)$, the deadzone-quadratic penalty function can be formulated as follows:

$$
\psi_{q}(x):=(\ln (1+\exp (x-z))+\ln (1+\exp (-x-z)))^{2} .
$$

The gradient of the deadzone-linear penalty function is a linear continuous function with a deadzone area, $[-z, z]$, as follows:

$$
\frac{d \psi_{q}(x)}{d x}=2 \psi_{l}(x) \frac{d \psi_{l}(x)}{d x} .
$$

For sake of simplicity, Fig. 2 shows the proposed $\psi_{q}(x)$ and $\psi_{l}(x)$ penalty functions for a scalar residual with $z=5$ in comparison with $\phi_{q}(x), \phi_{l}(x), \ell_{2}$, and $\ell_{1}$-norms. Note that when the state residual is within the zone, the gradient is non-zero and the optimality conditions are satisfied as $\ell_{1}$ and $\ell_{2}$-norms. In other words, the states will converge to final reference set-point values but slower than conventional norms which lead to the energy-efficient behaviour. 


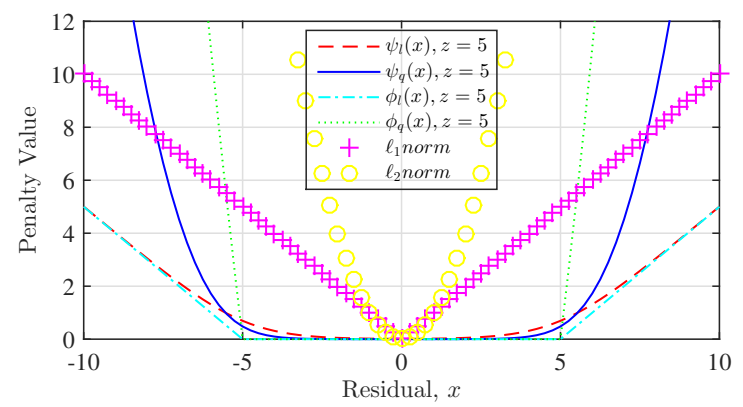

Fig. 2: Deadzone Penalty Functions with Relative Comparisons

\subsection{Case Study: Ext-Eco-CC Mathematical Optimisation Problem}

The state vector for the Ext-Eco-CC system from Eqs. (1), (2), and (4), is defined as $x_{t}=[s, v, e]^{T} \in \mathbb{R}^{3}$; the control input is the traction input applied on BEV, $u_{t}=u \in \mathbb{U} \subset \mathbb{R}$ (for more details see [6]); Please note that all states are measurable and the measurement noise is negligible.

The cost-per-stage function for Ext-Eco-CC system is defined as:

$$
J_{c}\left(\hat{x}_{i}, u_{i}\right):=\sum_{i=0}^{N-1} \frac{1}{2}\left[\psi_{q}\left(\hat{x}_{i}-x_{r e f}\right)_{Q}+\left\|u_{i}-u_{r e f}\right\|_{R}^{2}\right],
$$

with corresponding weights $(Q$ and $R)$. The final cost function for Ext-Eco-CC system is defined as:

$$
J_{f}\left(\hat{x}_{N}\right):=\frac{1}{2} \psi_{q}\left(\hat{x}_{N}-x_{r e f}\right)_{Q} .
$$

The lateral acceleration of the BEV should be lower than the comfort level $\left(\hat{\omega}_{r e f}\right)$ as inequality constraint as follows:

$$
g_{1}\left(\hat{s}_{i}, \hat{v}_{i}\right):=\hat{v}_{i}^{2} / f_{c r v}\left(\boldsymbol{\delta}\left(\hat{s}_{i}\right)\right) \leq \hat{\omega}_{r e f},
$$

The velocity of the BEV should also be lower than speed limit zones as:

$$
g_{2}\left(\hat{s}_{i}, \hat{v}_{i}\right):=\hat{v}_{i} \leq f_{l m t}\left(\hat{s}_{i}\right) .
$$

In addition, the velocity should be within the standstill and the reference set-point socalled funnel concept (see e.g., [15]) as follows:

$$
g_{3}\left(\hat{v}_{i}\right):=0 \leq \hat{v}_{i} \leq v_{r e f}+v_{r l x}
$$

where $v_{r e f}$ is the reference set-point and $v_{r l x}$ is the relaxed velocity for the inequality constraint. One of the efficient methods to solve the resulting OCP in the receding horizon manner can be based on Pontryagin's Minimum Principle. The obtained dual OCP can be solved efficiently in real-time by the Continuation and Generalised Minimal RESidual (C/GMRES) with a proper inequality constraints handling method (for more details see $[12,16])$. 


\section{System Evaluation}

The proposed Ext-Eco-CC system has been evaluated with practical experiments on a test track using realistic values of the parameters. A Smart Electric Drive third generation (Smart-ED) commercial BEV, which is available for practical experiments, is chosen here to model the dynamics of a BEV and its energy consumption (Fig.3). A

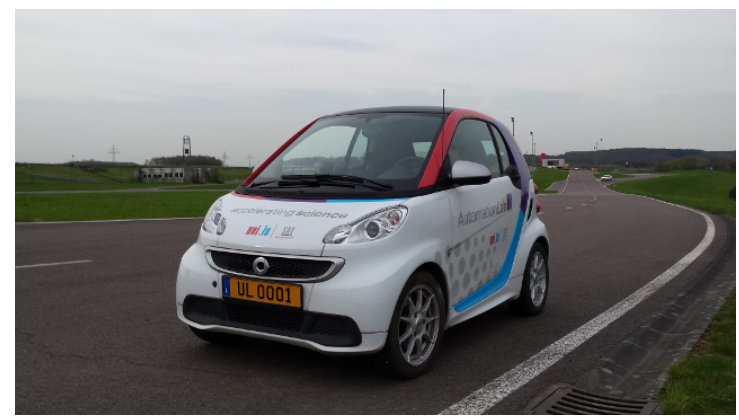

Fig. 3: Smart Fortwo Electric Drive

closed test track located at Colmar-Berg, Luxembourg, (CFC) is chosen to model the road geometry with traffic information (Fig. 4). The test track has a total length of

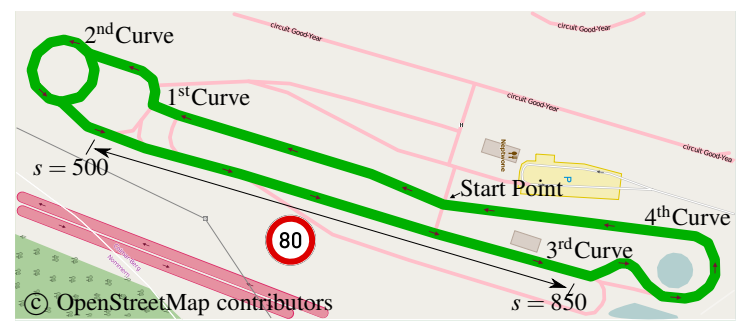

Fig. 4: Centre de Formation pour Conducteurs (CFC) [17]

$1.255 \mathrm{~km}$ and includes curves and relative slope profile. This track has four main curves with $20 \mathrm{~m}, 25 \mathrm{~m}, 15 \mathrm{~m}$, and $27 \mathrm{~m}$ radius. Note that the speed limit zone is not imposed in system evaluation in order to simplify the system evaluation (for more detail, see [6]).

\subsection{Experimental Setup}

In order to validate the proposed concept, the NMPC with deadzone-quadratic penalty function is experimentally implemented on the Smart-ED vehicle and the Ext-Eco-CC system is tested on the CFC test track. The position of the Smart-ED is updated by 
the Global Positioning System (GPS) sensor. The velocity and energy consumption of the vehicle including the battery current and voltage information is updated by the Controller Area Network (CAN-bus) through the On-Board Diagnose (OBD) interface. The onboard computational resource for the Ext-Eco-CC concept is foreseen by a Linux operating system on the Intel ${ }^{\circledR}$ Core $^{\mathrm{TM}}$ i7 with a memory of $7.7 \mathrm{GiB}$ PC and connection panel. The connection panel is developed for the system power supply and actuators communication (Fig. 5).

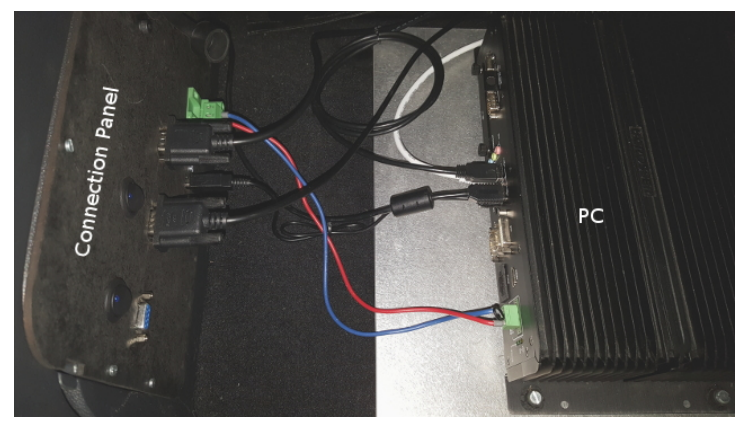

Fig. 5: The Linux operated PC with Connection Panel

The control input of the proposed NMPC with deadzone-quadratic penalty function is realised by actuating either the accelerator pedal or brake actuator. The accelerator pedal is replaced by an electronic board (E-accelerator) to manipulate the required acceleration and imitates the electric signals generated by the original accelerator pedal of the Smart-ED. The brake actuator is manipulated by an electric stepper motor that is connected to the brake pedal by a planetary gearbox and flexible cable. The automatic brake actuation is designed in a way that preserves the possibility for the driver to brake in emergency cases. Fig. 6 shows the configuration of the E-accelerator and brake actuators for the Ext-Eco-CC system.

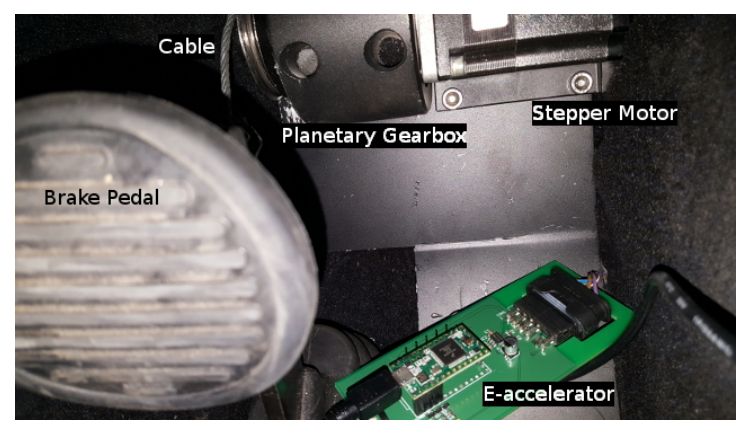

Fig. 6: Automatic Accelerator and Brake Actuators 


\subsection{Experimental Results Validation}

In order to show the performance of the proposed Ext-Eco-CC system, a prediction horizon for the predictive controller is set to $T=15 \mathrm{~s}$, to cover upcoming road geometry, and traffic speed limit zone with $N=30$ discretized steps. The constants in performance index function are set as $Q=\operatorname{diag}[0,2,0]$, and $R=\operatorname{diag}[450]$. Note the weight for energy-consumption is set to zero since the effectiveness of the deadzone-quadratic penalty function in energy efficiency is the main focus in this paper. The reference for the lateral acceleration comfort level is $\omega_{\text {ref }}=3.7 \mathrm{~m} / \mathrm{s}^{2}$.

We have compared our proposed deadzone-quadratic NMPC (DQ-NMPC) with the conventional NMPC with $\ell_{2}$-norm (C-NMPC) and human driver (HD) in terms of velocity regulation, travel time $(t)$, power consumption profile and total energyconsumption (e). For the sake of fair comparison, all of the tests started from the standstill and the maximum reference velocity value is chosen, $v_{r e f}=100 \mathrm{~km} / \mathrm{h}$ without imposing speed limit zone. The desired reference zone for velocity tracking is chosen as $z=2 \mathrm{~m} / \mathrm{s}$. We have proposed human driver to drive as fast and energy-efficient as possible.

Fig. 7a shows the performance of various tests in terms of velocity regulations and total travel time. The DQ-NMPC and C-NMPC increase the velocity up to reaching the first curve $(220 \leq s \leq 270)$ where the lateral acceleration constraint should be satisfied. As it is shown, the human driver is faster than the controllers. However, during the first and second curves $(320 \leq s \leq 440)$, the controllers and human drivers show similar behaviour. Afterwards, the controllers increase over again the velocity up to the point the third curve $(860 \leq s \leq 930)$ are in their prediction horizon. This leads to the beginning of slowing down predictively to satisfy the upcoming constraints in an energy-efficient way. The human driver show late but sharper velocity reduction which may not be an energy-efficient technique. Finally, the controllers keep the velocity during the fourth curve $(930 \leq s \leq 1045)$ and speed up once more to reach the starting point on the test track. Thus, the presented result shows that the maximum reference velocity is not reachable, however, the reference velocity for less than $v_{r e f}=80 \mathrm{~km} / \mathrm{h}$ is reachable on the experimental tests carried on the CFC track. Fig. 7b shows the power consumption profile and total energy. Note that negative power consumption refers to energy recovery mechanism.

Fig. 8a and Fig. 8b show the velocity and power consumption normalised histogram information. The proposed DQ-NMPC benefits from an improved penalty function which leads to a denser velocity and power consumption distribution compared to the C-NMPC and human driver. Based on achieved results, it is shown that the set-point value is not reachable on the test track by the controllers or the human driver. The DQNMPC leads to more steady velocity profile and consequently the better drive comfort with relatively small increased travel time. The total energy consumption of DQ-NMPC is $+13.65 \%$ more energy efficient than the human driver and $+6.58 \%$ more energy efficient than the C-NMPC. In other words, for longer trips with more hilly and curvy roads, our proposed method has higher potential to be more energy-efficient. It is noteworthy that the OCP average calculation time for the DQ-NMPC is $2.35 \mathrm{~ms}$ which indicate its real-time capability of the proposed controller. 
(a)

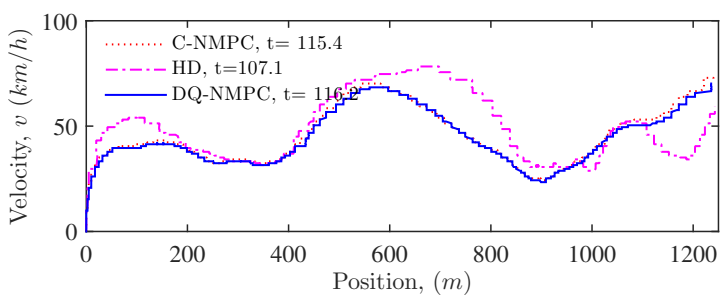

(b)

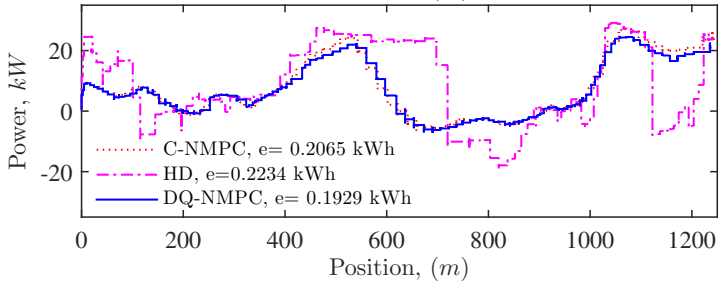

Fig. 7: Experimental results of DQ-NMPC in compare with C-NMPC and Human Driver for (a) Velocity regulation, and (b) Power consumption profile.

(a)
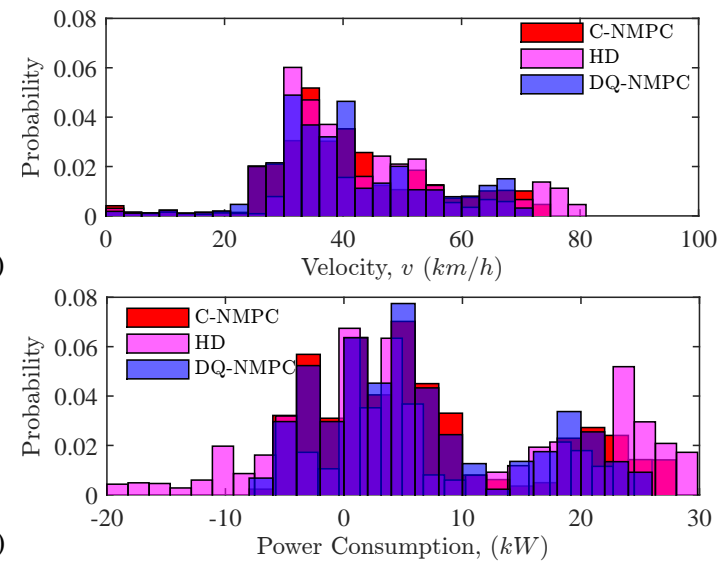

Fig. 8: Experimental performance distribution of DQ-NMPC in compare with C-NMPC and the Human Driver for (c) Velocity and (d) Power consumption.

\section{Conclusion and Future Research}

A semi-autonomous ecological driver assistance system was developed to regulate the velocity in an energy-efficient manner for the electric vehicles. A real-time nonlinear receding horizon optimal controller with approximate deadzone-quadratic penalty function was proposed to plan online the cost-effective velocity profile. The limited cruising range of the electric vehicles was improved by the assessed low penalty value on set-point tracking zone and ecological driving techniques. Various tests on a semiautonomous electric vehicle in terms of real-time states regulation and constraints fulfilment were carried out. The effectiveness of the proposed method was demonstrated by 
the achieved field experimental results. Further practical experiments will be conducted including extending the functionalities of semi-autonomous ecological driving.

\section{References}

1. Ehsani, M., Gao, Y., Emadi, A.: Modern Electric, Hybrid Electric, and Fuel Cell Vehicles: Fundamentals, Theory, and Design, 2, illustr edn. CRC Press (2009)

2. Li, N.I., He, C.R., Orosz, G.: Sequential Parametric Optimization for Connected Cruise Control with Application to Fuel Economy Optimization. In: 2016 IEEE 55th Conference on Decision and Control (CDC), pp. 227-232. IEEE, Las Vegas, NV, USA (2016). DOI 10.1109/CDC.2016.7798274

3. Schwickart, T., Voos, H., Hadji-Minaglou, J.R., Darouach, M., Rosich, A.: Design and simulation of a real-time implementable energy-efficient model-predictive cruise controller for electric vehicles. Journal of the Franklin Institute 352(2), 603-625 (2015). DOI 10.1016/j.jfranklin.2014.07.001

4. Schwickart, T., Voos, H., Hadji-Minaglou, J.R., Darouach, M.: A Fast Model-Predictive Speed Controller for Minimised Charge Consumption of Electric Vehicles. Asian Journal of Control 18(1), 133-149 (2016). DOI 10.1002/asjc.1251

5. Kamal, M.a.S., Mukai, M., Murata, J., Kawabe, T.: Ecological Vehicle Control on Roads With Up-Down Slopes. IEEE Transactions on Intelligent Transportation Systems 12(3), 783-794 (2011). DOI 10.1109/TITS.2011.2112648

6. Sajadi-alamdari, S.A., Voos, H., Darouach, M.: Nonlinear Model Predictive Extended EcoCruise Control for Battery Electric Vehicles. In: 24th Mediterranean Conference on Control and Automation (MED), pp. 467-472. IEEE, Athens (2016). DOI 10.1109/MED.2016. 7535929

7. Saerens, B., Rakha, H., Diehl, M., Van den Bulck, E.: A methodology for assessing ecocruise control for passenger vehicles. Transportation Research Part D: Transport and Environment 19, 20-27 (2013). DOI 10.1016/j.trd.2012.12.001

8. Sciarretta, A., De Nunzio, G., Ojeda, L.L.: Optimal Ecodriving Control: Energy-Efficient Driving of Road Vehicles as an Optimal Control Problem. IEEE Control Systems 35(5), 71-90 (2015). DOI 10.1109/MCS.2015.2449688

9. Boyd, S., Vandenberghe, L.: Convex Optimization. Cambridge University Press (2004)

10. Gros, S., Zanon, M.: Penalty Functions for Handling Large Deviation of Quadrature States in NMPC. IEEE Transactions on Automatic Control 9286(c), 1-1 (2017). DOI 10.1109/ TAC.2017.2649043

11. Eskandarian, A. (ed.): Handbook of Intelligent Vehicles, vol. 2. Springer London, London (2012). DOI 10.1007/978-0-85729-085-4

12. Ohtsuka, T.: A continuation/GMRES method for fast computation of nonlinear receding horizon control. Automatica 40(4), 563-574 (2004). DOI 10.1016/j.automatica.2003.11.005

13. LeCun, Y., Bengio, Y., Hinton, G.: Deep learning. Nature 521(7553), 436-444 (2015)

14. Dugas, C., Bengio, Y., Bélisle, F., Nadeau, C., Garcia, R.: Incorporating Second-order Functional Knowledge for Better Option Pricing. In: Proceedings of the 13th International Conference on Neural Information Processing Systems, NIPS'00, pp. 451-457. MIT Press, Cambridge, MA, USA (2000)

15. Maciejowski, J.M.: Predictive Control: With Constraints. Pearson Education. Prentice Hall (2002)

16. Huang, M., Nakada, H., Butts, K., Kolmanovsky, I.: Nonlinear Model Predictive Control of a Diesel Engine Air Path: A Comparison of Constraint Handling and Computational Strategies. IFAC-PapersOnLine 48(23), 372-379 (2015). DOI 10.1016/j.ifacol.2015.11.308

17. Centre de Formation pour Conducteurs. URL http://www.cfc.lu/ 